\title{
Mitral valve prolapse
}

\author{
Mostly benign
}

In the more than 30 years since Reid and Barlow et al proposed that mid to late systolic clicks and apical late systolic murmurs were of mitral valvular origin ${ }^{12}$ and the 27 years since Criley and colleagues coined the term "mitral valve prolapse" to describe posterior mitral leaflet motion in systole ${ }^{23}$ there has been an explosion of interest in the condition. The explanation for this sustained high level of interest in mitral valve prolapse, a disorder that is often asymptomatic and usually clinically benign, lies in its high prevalence (particularly in young people), non-specific clinical presentation, and potential (albeit low) for serious clinical complications in otherwise healthy people.

Mitral valve prolapse is the most common cardiac valvular abnormality in Britain and the United States (if one excludes aortic sclerosis). ${ }^{4}$ Its reported prevalence ranges from $4 \%$ to $17 \%$ in women and $2 \%$ to $12 \%$ in men. ${ }^{4}$ With increasing age the prevalence falls in women but remains unchanged in men. ${ }^{4}$ Differences in age, gender distribution, diagnostic criteria, and diagnostic techniques account for the wide disparity of prevalences among studies. ${ }^{4}$ During the past decade substantial progress has been made in clarifying the diagnostic criteria. ${ }^{56}$ and defining secondary causes ${ }^{7-9}$ (more than $98 \%$ of cases are primary). Most recently, however, research has focused on the pathogenesis of non-specific symptoms ${ }^{7-12}$ and the natural history of the condition. ${ }^{13-17}$

Most people with mitral valve prolapse are asymptomatic and do not come to medical attention. ${ }^{7}$ If they do it is usually for evaluation of non-specific cardiac or neurological symptoms or for symptoms referrable to complications. Some cases are detected incidentally during cardiac auscultation or echocardiography for evalution of a systolic murmur or suspected unrelated cardiac disease. ${ }^{7}$

Non-specific symptoms associated with mitral valve prolapse are chest pain, palpitations, pre-syncope or syncope, and effort intolerance (characterised by dyspnoea and lassitude). ${ }^{7}$ The term "mitral valve prolapse syndrome" is now customarily used to differentiate prolapse associated with non-specific symptoms from anatomical prolapse. ${ }^{?}$

Chest pain is the most common non-specific symptom, occurring in $60 \%$ of those with the syndrome. ${ }^{710}$ It is usually precordial, often left sided, and characterised as sharp, sticking, or lancinating. Occasionally it has an angina-like quality. ${ }^{70}$ Attempts to attribute chest pain to myocardial ischaemia have been unrewarding. ${ }^{10}$ Mitral valve prolapse occurs disproportionately frequently in subjects with various causes of chest pain including coronary artery spasm, oesophageal spasm, panic disorder, and chest wall pain. ${ }^{10}$ Thus, while a specific (but as yet undefined) mechanism of chest pain may exist in patients with the mitral valve prolapse syndrome, ${ }^{7}$ other conditions may coexist and be responsible for chest pain in a given patient.

Palpitations occur in about a half of people with the syndrome. ${ }^{7}$ Although various atrial and ventricular arrhythmias may account for palpitations in some patients, there is often discordance between reported symptoms and the presence and timing of arrhythmias during ambulatory or patient activated electrocardiographic monitoring. ${ }^{7}$ Many patients with mitral valve prolapse report palpitations during acceleration of normal sinus rhythm, suggesting that increased nociception may play a part.?

Pre-syncope and syncope may result from tachyarrhythmias (for example, ventricular tachycardia) but more commonly occur without disturbances of rhythm. ${ }^{7}$ Postural hypotension and vasodepressor responses occur commonly.

The pathogenesis of effort intolerance in the mitral valve prolapse syndrome is unknown. Many attempts to associate mitral valve prolapse with panic syndromes have produced conflicting information. A recent controlled study suggested a high rate of co-occurrence but no cause and effect relation between mitral valve prolapse and panic syndromes. ${ }^{11}$

An impressive body of evidence has accumulated to suggest the presence of dysautonomia in many patients with the mitral valve prolapse syndrome. ${ }^{12}$ Various investigators have reported exaggerated heart rate and blood pressure responses to stimuli (including vasodepressor responses), postural hypotension, high circulating catecholamine concentrations, hyperresponsiveness to infused catecholamines (with provoction of chest pain and other symptoms in some cases), abnormal catecholamine regulation and baroreflex modulation, decreased intravascular and intraventricular volume on standing, and activation of atrial natriuretic peptide. ${ }^{12}$ These findings have led Boudoulas and Wooley to propose that the syndrome may in part be a neuroendocrinopathy. ${ }^{12}$

If alternative causes of non-specific symptoms are not identified it would seem prudent to attribute such symptoms to mitral valve prolapse and start empirical treatment. Patients with the mitral valve prolapse syndrome and mild symptoms frequently respond to reassurance. $\beta$ Blockers may produce improvement in chest pain in $25-50 \%$ of cases, and severe palpitations often respond well to $\beta$ blockade whether or not they are caused by arrhythmias. Patients with presyncope or syncope that remains unexplained after non- 
invasive cardiac evaluation should be referred for invasive electrophysiological studies. Patients with vasodepressor syncope and mitral valve prolapse may respond to $\beta$ adrenergic blockade. Effective treatment of the effort syndrome remains difficult. Symptoms of the syndrome often occur in clusters during periods of increased emotional or physical stress; in such people $\beta$ blockers may be targeted at symptom peaks rather than given regularly.

Between $3 \%$ and $10 \%$ of people with symptomatic mitral valve prolapse develop potentially life threatening complications. These are congestive heart failure due to progressive mitral regurgitation or mitral regurgitation associated with ruptured chordae tendineae, infective endocarditis, cerebral embolism, and sudden death. Studies by Marks and others indicate that many of these complications occur most commonly in patients with thickened mitral valve leaflets and are more likely to occur in older men with prolapse. ${ }^{13-15}$

Congestive heart failure due to severe mitral regurgitation associated with myxomatous degeneration and mitral valve prolapse may respond for a time to treatment with diuretics and vasodilators but is now the commonest indication for mitral valve replacement in elderly people and for mitral annuloplasty at any age..$^{13-15}$ Flail mitral leaflet virtually always requires mitral valve replacement or repair. ${ }^{13-15}$

Infective endocarditis has been reported to occur in $2-8 \%$ of patients with known mitral valve prolapse. ${ }^{13-16}$ Moreover, retrospective analyses indicate that mitral valve prolapse was present in up to one third of cases of documented infective endocarditis. ${ }^{16}$ Important risk factors for infective endocarditis in patients with mitral valve prolapse are the presence of mitral regurgitation and thickened (myxomatous) mitral leaflets. ${ }^{13-16}$ The pathogenesis, clinical features, prognosis, and therapeutic implications of infective endocarditis associated with mitral valve prolapse are similar to those associated with other forms of mitral valve disease. ${ }^{13-16}$ Antibiotic prophylaxis should probably be reserved for those with mitral regurgitation or thickened mitral leaflets, or both. ${ }^{13-16}$

Cerebral embolism occurs with a disproportionately high frequency in young people with mitral valve prolapse and probably in older people as well. ${ }^{13-15} 17$ Thrombi have been detected on mitral valve leaflets of patients with mitral valve prolapse who have died of cerebral embolism. ${ }^{17}$ Advanced age seems to be the main risk factor. ${ }^{13-1517}$ Cerebral embolism seems to occur with equal frequency in those with normal and thickened mitral valve leaflets. Shortened platelet survival time and increased platelet coagulant activity have been reported in studies of patients with cerebral embolism and mitral valve prolapse, ${ }^{17}$ but whether this is a cause or effect of thrombosis and embolism is uncertain. The efficacy of antiplatelet therapy in preventing thromboembolism is unproved, but it is often used empirically. Warfarin should be used in patients who have suffered previous thromboembolic episodes. Sudden death in patients with mitral valve prolapse is extremely rare and is usually due to sustained ventricular tachycardia or ventricular fibrillation. ${ }^{13-15}$ In addition to thickened mitral valve leaflets, the presence of delayed repolarisation is a risk factor for sudden death. Treatment should be guided by invasive electrophysiological testing in people with mitral valve prolapse who have had ventricular tachyarrhythmias.

MARTIN A ALPERT

University of South Alabama Medical Centre, Director, division of cardiology

Mobile, AL 36617-2293,

USA

1 Reid JV. Mid-systolic clicks. $S$ Afr Med $\mathcal{f} 1961$;35:353-5.

2 Barlow JB, Pocock WA, Marachand P, Denny M. The significance of late systolic murmurs. Am Hear $\mathcal{F}$ 1963;66:443-52

3 Criley JM, Lewis KM, Humphries JO, Ross RS. Prolapse of the mitral valve: clinical and cineangiographic findings. $\mathrm{Br}$ Hear $f$ 1966;28:488-96.

4 Boudoulas H, Wooley CF. Mitral valve prolapse: prevalence. In: Boudoulas $\mathrm{H}$, Wooley CF, eds. Mitral valve prolapse and the mitral valve prolapse syndrome. Mount Kisco, NY: Futura Publishing, 1988:161-70.

5 Perloff JK, Child JE, Edwards DE. New guidelines for the clinical diagnosis of mitral valve prolapse. Am f Cardiol 1986;57:1124-9.

6 Devereux RB, Kramer-Fox R, Kligfield P. Mitral valve prolapse: causes, clinical manifestations and management. Ann Intern Med 1989;111:305-17.

7 Boudoulas H, Wooley CF. Mitral valve prolapse: clinical presentation, diagnostic evaluation and therapeutic considerations. In: Boudoulas $\mathrm{H}$, Wooley CF, eds. Mitral valve prolapse and mitral valve prolapse syndrome. Mount Kisco, NY: Futura Publishing, 1988:299-344.

8 King BD, Clark MA, Bobo N, Kilman JW, Wooley CF: "Myxomatous" mitral valves: collagen disolution as the primary defect. Circulation 1982;66:288-96.

9 Barlow DB. Perspectives on the mitral valve. Philadelphia: FA Davis, 1987:61.

10 Alpert MA, Mukerju V, Sabeti M, Russell J, Beitman BD. mitral valve prolapse, panic disorder and chest pain. Med Clin North Am 1991;75:1119-33.

11 Alpert MA, Sabeti M, Kushner MG, Beitman BD, Russell JL, Thiele JR, et al. Frequency of isolated panic attacks and panic disorder in patients with mitral valve prolapse. $A m$ f Cardio 1992;69:1489-90.

12 Boudoulas $\mathrm{H}$, Wooley CF. Hyperadrenergic state, autonomic dysfunction, and pathogenesis of symptoms in mitral valve prolapse syndrome. In: Boudoulas $\mathrm{H}$, Wooley CF, eds. Mitral valve prolapse and the mitral valve prolapse syndrome. Mount Kisco, NY: Futura Publishing, 1988: prolapse and

13 Kolibash AJ Jr. Natural history of mitral valve prolapse. In: Boudoulas H, Wooley CF, eds. Mitral valve prolapse and the mitral valve prolapse syndrome. Mount Kisco, NY: Futura Publishing, 1988:257-75.

14 Devereux RB, Hawkins I, Kramer-Fox R, Lutas EM, Hammond IW, Spitzer MC, et al. Complications of mitral valve prolapse. Disproportionate occurrence in men and older patients. Am F Med 1986;81:751-8.

15 Marks AR, Choong CU, Sanfillipo AJ. Identification of high risk and low risk subgroups of patients and mitral valve prolapse. $N$ Engl $f$ Med 1989;320:1031

16 MacMahon SW, Hickey AJ, Wilcken DE, Wattes JT, Feneley MR, Hickie JB. Risk of infective endocarditis in mitral valve prolapse with and without precordial systolic murmurs. Am f Cardio 1986;59:105-8.

17 Lewis RP. Cerebral embolism in mitral valve prolapse. In: Boudoulas H, Wooley CF, eds. Mitral valve prolapse and the mitral valve prolapse syndrome. Mount Kisco, NY: Futura Publishing, 1988.289-98.

\section{What social workers need}

\section{$A$ General Social Services Council, better education, and a wider range of employers}

Social work has been harshly judged during the past 20 years on some spectacular failures in child protection. Its successes -monitoring 45000 children on child protection registers and helping to halve the number of children in public careare largely unreported. Its achievements with adults are overshadowed by current preoccupations with children's issues. Action is now needed on three fronts to enable social workers to flourish: the professional institutions of social work, education and training, and the agencies that employ social workers.

Social workers engage with important, sensitive, and private aspects of the lives of individual people and families. Their work directly affects hundreds of thousands of citizens. Yet social work is not regulated by an independent body: qualifications for appointment (except in the case of approved social workers) and arrangements for discipline are matters for the employer alone. A proposal to remedy this by establishing a General Social Services Council is currently before the secretary of state. At little or no cost to the public purse the council would regulate the conduct of individual practitioners and set standards of competence for education and training. Social workers in residential and community 